\title{
Experiencia del cuidador familiar ante la muerte del niño con cáncer*
}

\author{
Sonia Carreño-Moreno - Lorena Chaparro-Díaz $^{b}$

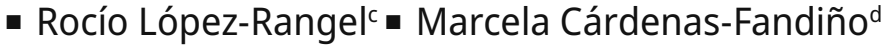 \\ - Mabel Carrillo-González ${ }^{e}$ - Olga Gómez-Ramírez
}

\begin{abstract}
Resumen: En este estudio se trazó como objetivo la descripción de la experiencia del cuidador familiar ante la muerte del niño con cáncer. Para ello, se utilizó como método un estudio cualitativo con enfoque fenomenológico descriptivo bajo la perspectiva de Colaizzi, en el que se incluyeron 18 participantes que cumplieron con los criterios de inclusión de ser padre o madre de un niño con cáncer que haya fallecido en un periodo de 5 meses a 5 años. Se realizaron entrevistas a profundidad hasta lograr saturación teórica. Como resultados, se obtuvieron seis temas que describen la experiencia del cuidador: idealización del niño fallecido, batalla perpetua, vínculo inquebrantable, pérdida de sentido, levantarse y seguir, y lucha espiritual; estos representan la percepción y vivencia de los cuidadores en el duelo. Como conclusiones se consideró que las expresiones de dolor, incertidumbre y vacío son sentimientos y sensaciones descritas por los cuidadores, que hacen parte del proceso de duelo, pero también describen su paso hacia la aceptación y trascendencia, en la que dan importancia al ser espiritual del niño como una forma de creer que sigue junto a ellos.
\end{abstract}

* Artículo de investigación.

a Doctora en Enfermería, Magíster en Enfermería, Enfermera. Profesora Universidad Nacional de Colombia, Facultad de Enfermería, Bogotá, Colombia. Correo electrónico: spcarrenom@unal.edu.co ORCID •

b Doctora en Enfermería, Enfermera. Profesora Universidad Nacional de Colombia, Facultad de Enfermería, Bogotá, Colombia.

Correo electrónico: olchaparrod@unal.edu.co ORCID •

c Magíster en Enfermería, Enfermera. Instituto Nacional de Cancerología, Bogotá, Colombia. Correo electrónico: rociolopez_1603@hotmail.com ORCID •

d Magíster en Enfermería, Enfermera. Instituto Nacional de Cancerología, Bogotá, Colombia. Correo electrónico: mcardenasf@cancer.gov.co ORCID •

e Doctora en Enfermería, Magíster en Enfermería, Enfermera. Profesora Universidad Nacional de Colombia, Facultad de Enfermería, Bogotá, Colombia. Correo electrónico: gmcarrillog@unal.edu.co ORCID •

f Doctora en Enfermería, Magíster en Enfermería, Enfermera. Profesora Universidad Nacional de Colombia, Facultad de Enfermería, Bogotá, Colombia.

Correo electrónico: ojgomezr@unal.edu.co ORCID • 
Recibido: 26 de diciembre de 2018 Aceptado: 21 de febrero de 2019

Disponible en línea: 15 de septiembre de 2020

Cómo citar: Carreño-Moreno S, Chaparro Díaz L, López-Rangel R, Cardenas-Fandiño M, CarrilloGonzález M, Gómez-Ramírez O. Experiencia del cuidador familiar ante la muerte del niño con cáncer. Rev. latinoam. bioet [Internet]. 15 de septiembre de 2020; 20(1). Disponible en: https://doi. org/10.18359/rlbi.3841

Palabras clave: duelo; cuidadores; niño; cáncer; profesionales de la salud

\section{Relative caregiver experience facing the death of a child with cancer}

Abstract: The objective of this study is to describe the relative caregiver's experience when facing the death of a child with cancer. With this purpose, the Colaizzi's descriptive phenomenological method was used in a qualitative study including 18 participants meeting the inclusion criteria of being parent to a child with cancer who died in a period from 5 months to 5 years. In-depth interviews were performed until reaching theoretical saturation. As a result, 6 topics describing the caregivers experience were found: idealization of the sick child, perpetual battle, unbreakable bond, loss of sense, get up and move on, and spiritual struggle; representing the perception and experience of caregivers in mourning. It was concluded that expressions of pain, uncertainty and emptiness are feelings and sensations described by the caregivers, which are part of the mourning process, but also describe their movement to acceptance and transcendence, where they give importance to the spiritual being of the child as the way to believe cheats still next to them.

Keyword: mourning; caregivers; child; cancer; health professionals

\section{Experiência do cuidador familiar ante a morte da criança com câncer}

Resumo: O objetivo deste estudo é descrever a experiência do cuidador familiar ante a morte da criança com câncer. Para isso, foi utilizado como método o estudo qualitativo com abordagem fenomenológica descritiva sob a perspectiva de Colaizzi, no qual foram incluídos 18 participantes que cumpriram com os critérios de inclusão: ser pai ou mãe de criança com câncer que tivessem falecido em um período de 5 meses a 5 anos. Foram realizadas entrevistas a profundidade até conseguir saturação teórica. Como resultados, foram obtidos seis temas que descrevem a experiência do cuidador: idealização da criança falecida, batalha perpétua, vínculo inquebrantável, perda de sentido, seguir adiante e luta espiritual; estes representam a percepção e vivência dos cuidadores no luto. Como conclusões, foi considerado que as expressões de dor, a incerteza e o vazio são sentimentos e sensações que os cuidadores descrevem como parte do processo de luto, mas também como passagem à aceitação e à transcendência, na qual dão importância ao ser espiritual da criança como forma de acreditar que continua junto deles.

Palavras-chave: luto; cuidadores; criança; câncer; profissionais da saúde 


\section{Introducción}

De acuerdo con la Organización Mundial de la Salud (OMS), cada año más de 150.000 niños son diagnosticados con cáncer, y cerca de un $90 \%$ de ellos no sobrevive debido a que viven en países con recursos limitados, lo que dificulta recibir o completar el tratamiento (1). En Colombia, Piñeros reveló que la mortalidad en niños por esta causa ascendió del 2,2\% en 1985, al 3,5\% en 2008, hecho explicado por las dinámicas económicas y la transición demográfica del país (2).

Sumado a estas cifras, el proceso de enfermedad crónica y la potencial amenaza a la vida de un niño genera en los padres sentimientos de incertidumbre e intensa aflicción, que son descritos por algunos como el mayor evento estresante de su ciclo vital (3), puesto que para un padre esto simboliza la acción reversa del orden natural de la vida y acaba intempestivamente con los deseos y el proyecto de vida de su hijo (4).

El proceso de duelo en el caso de cuidadores de niños con cáncer suele ser prolongado y asociado con manifestaciones de tipo somático y emociones negativas. La mayoría de cuidadores familiares de niños con cáncer que reciben orientación y apoyo logran afrontar el duelo y continuar con su vida diaria luego de 18 meses posteriores a su muerte (4); sin embargo, en algunos casos en los que no se realiza seguimiento, este proceso puede tomar de 4 a 9 años (3), y tener implicaciones negativas en el estilo de vida como el alcoholismo y el tabaquismo, además de cambios psicopatológicos (5).

El acompañamiento a padres de niños con cáncer por parte de los profesionales de la salud suele ser difícil, sobre todo por considerarse una realidad atemorizante que demanda conocimiento de la situación y capacitación específica para el manejo de los aspectos psicológicos y emocionales para enfrentar las necesidades de los familiares, la agonía, el dolor y finalmente la muerte de los pacientes (6), por ello resulta fundamental comprender a profundidad la experiencia del duelo de cuidadores de niños con cáncer.

Ante este panorama incierto, los cuidadores de niños con cáncer viven en un continuo proceso de duelo hasta que se produce la muerte del niño
$(7,8,9)$. Además, es una experiencia única y que cada cuidador describe de diferentes maneras. Por ello, la comprensión de las necesidades de cuidado en la vivencia del duelo del cuidador familiar del niño fallecido por cáncer tiene un alto impacto en esta experiencia, dado el vínculo y cercanía adquiridos entre el cuidador y profesionales de la salud en enfermería, medicina y psicología.

Para diversos autores $(10,11,12,13)$, el proceso de duelo de los padres puede llegar a ser un proceso complicado por factores como la relación de parentesco, ya que esta pérdida ha sido reconocida como la más intensa y duradera, con graves consecuencias en todos los ámbitos de su vida, en especial la relacional y la laboral.

El proceso de duelo en los padres se encuentra desbordado de reacciones emocionales como la ira, la incredulidad, el miedo y la culpa. Esta última es una de las más reconocidas e identificadas en ellos $(14,15)$, por lo que muchos estudios la reconocen como causa de mayor ansiedad, aislamiento y depresión $(16,17)$. Por ello, es importante el acompañamiento a estos padres, así como la posibilidad de brindar soporte social, no solo a través de intervenciones terapéuticas para el duelo, sino contactando con redes o grupos de apoyo de otros cuidadores que atraviesan por situaciones similares, ya que el compartir la experiencia con pares les permite no sentirse solos en el proceso $(11,18)$.

Con las expresiones propias de los cuidadores ante un proceso tan doloroso como la pérdida de un hijo debido al cáncer, los profesionales de la salud tienen el deber ético de escuchar, acompañar e intervenir para que el duelo tenga una resolución satisfactoria, de manera que se hace relevante conocer la voz de los protagonistas, sus percepciones $y$ vivencias, a fin de identificar sus necesidades (6).

En síntesis, el objetivo de este estudio fue conocer la experiencia del duelo del cuidador familiar del niño fallecido por cáncer, lo que permite describir con amplitud las percepciones y vivencias de este proceso, y entender como experiencia la forma en que el ser humano razona e interpreta el mundo y lo que vive en él (19). La descripción de este fenómeno requiere de una profundidad que permita reconocer las particularidades de la experiencia, además de ser un insumo importante para 
el conocimiento en las ciencias de la salud y focalizar las intervenciones a esta población.

\section{Materiales y método}

Este es un estudio de abordaje cualitativo con enfoque de fenomenología descriptiva que pretende captar la esencia de las experiencias vividas de los cuidadores familiares de niños con cáncer que han fallecido (20). La fenomenología descriptiva permite entender a las personas que son potenciales sujetos de atención de los profesionales de la salud, para saber cuáles son sus reacciones, sentimientos y experiencias, y comprender sus necesidades.

Los sujetos de investigación cumplieron con los siguientes criterios de inclusión: 1) ser padre o madre de un niño fallecido por cáncer; 2) haber transcurrido un periodo de tiempo de 5 meses a 5 años después de la muerte del niño; 3) residir en Bogotá en el periodo posterior a la muerte del niño, y 4) ser mayor de 18 años. Se excluyeron los cuidadores de niños hospitalizados en el momento del estudio.

Como estrategia de recolección se utilizó la entrevista a profundidad (21), la cual fue considerada como adecuada para explorar información que resultara significativa para los participantes respecto a la elaboración de su proceso de duelo. Para este tipo de entrevista se creó un ambiente de confianza que les permitiera expresar sus ideas de forma libre, por lo que no se emitieron juicios sobre sus ideas o sentimientos expuestos. Como principio ético de no maleficiencia se utilizaron estrategias terapéuticas para el manejo de las emociones generadas por la vivencia y se buscó que el abordaje de la entrevista tuviera un aspecto terapéutico, pues se trata de una experiencia difícil y dolorosa para los participantes (22). El muestreo teórico fue utilizado como método de recopilación de datos (23).

Los datos recolectados fueron transcritos en su totalidad y de manera literal. Las entrevistas fueron almacenadas en el software Atlas-ti, licenciado por la Universidad Nacional de Colombia. Para realizar el análisis se utilizó el enfoque de Colaizzi (23), del cual se tomaron los siguientes pasos:

1. Definición del fenómeno de interés.

2. Recolección de las descripciones sobre este fenómeno.
3. Lectura de todas las descripciones de los participantes.

4. Volver a las transcripciones originales y extraer enunciados significativos.

5. Tratar de escribir el significado de cada enunciado significativo.

6. Organizar los significados formalizados en grupos de temas.

7. Escribir una descripción exhaustiva.

8. Volver a los participantes para validación de esta descripción.

9. En el caso de que los nuevos datos sean revelados durante la validación, incorporarlos a la descripción exhaustiva (23).

Las entrevistas fueron realizadas por dos de las investigadoras y el análisis por otras dos que no tuvieron contacto con los participantes. Después del primer análisis, se realizó una triangulación con el equipo investigador $y$, posteriormente, con los participantes, para validar la información. Con este proceso se dio cumplimiento a los criterios de rigor, credibilidad, reflexividad, neutralidad, consistencia y relevancia, necesarios para mantener un orden metodológico (24).

En el análisis, todas las entrevistas fueron leídas, con la idea de que los investigadores identificaran sus propios sentimientos ante el fenómeno de la experiencia del cuidador relacionados con la muerte del niño con cáncer. Después, en cada una de las entrevistas se seleccionaron las oraciones más significativas que describían el fenómeno. Estas oraciones se organizaron en doscientos códigos nominales, los cuales se agruparon en seis temas que surgieron a partir de descripciones realizadas por los entrevistados, por lo que se trató de mantener una relación directa entre la transcripción y el tema emergente. Por último, se realizó la descripción exhaustiva de cada tema.

Esta investigación tuvo aprobación del Comité de Ética y de Investigación del Instituto Nacional de Cancerología y dio cumplimiento a los lineamientos del Consejo Internacional Organizaciones de Ciencias Médicas (25) y la legislación colombiana de investigación en salud bajo la Ley 911 de 2004 
(26). Todos los participantes aceptaron participar de forma voluntaria, firmaron el consentimiento informado y se resolvieron dudas al respecto.

\section{Resultados}

En la Tabla 1 se describe la caracterización de los 18 participantes junto con los datos de los 18 niños a los que se les brindaba cuidado. Los cuidadores, que en su mayoría eran madres y padres de los niños, estaban entre los 19 y 69 años, con una media de 38.8 años, de los cuales el $88.9 \%$ son mujeres. De los niños fallecidos, la edad mínima fue de 6 meses y máximo de 18 años, con un promedio de 10 años, entre los cuales el 50\% tenía como diagnóstico oncológico algún tipo de Leucemia.

Tabla 1. Caracterización de los participantes

\section{Característica}

Datos del niño

Diagnóstico oncológico

\begin{tabular}{|c|c|}
\hline & $\underline{\text { Linfoma linfoblástico }}$ \\
\hline \multirow[t]{2}{*}{ Género } & Masculino \\
\hline & Femenino \\
\hline \multicolumn{2}{|l|}{ Datos del cuidador familiar } \\
\hline \multirow[t]{2}{*}{ Género } & Masculino \\
\hline & Femenino \\
\hline \multirow[t]{3}{*}{ Nivel de escolaridad } & Primaria \\
\hline & Bachillerato \\
\hline & Técnico y/o profesional \\
\hline \multirow[t]{4}{*}{ Procedencia } & Bogotá D.C. \\
\hline & Cundinamarca \\
\hline & Boyacá \\
\hline & Otros lugares del país \\
\hline \multirow[t]{3}{*}{ Estado civil } & Casado(a) \\
\hline & Soltero(a) \\
\hline & Unión Libre \\
\hline \multirow[t]{2}{*}{ Religión } & Católica \\
\hline & Cristiana \\
\hline \multirow[t]{2}{*}{ Único cuidador } & $\underline{\mathrm{Si}}$ \\
\hline & No \\
\hline
\end{tabular}

\section{Estadística descriptiva}

Edad del niño en el momento del fallecimiento (años)

Tiempo que lleva con la enfermedad el niño (años)

Edad del cuidador (años)

Tiempo que lleva como cuidador (años)
Porcentaje \% ( $n=18)$

\begin{tabular}{ll}
\hline 44.4 \\
\hline 16.6 \\
\hline 16.6 \\
\hline 16.6 \\
\hline 5.5 \\
\hline 50 \\
\hline 50
\end{tabular}

\begin{tabular}{|c|c|c|c|}
\hline \multicolumn{4}{|c|}{11} \\
\hline \multicolumn{4}{|c|}{89} \\
\hline \multicolumn{4}{|c|}{50} \\
\hline \multicolumn{4}{|c|}{38.8} \\
\hline \multicolumn{4}{|c|}{11.1} \\
\hline \multicolumn{4}{|c|}{5.5} \\
\hline \multicolumn{4}{|c|}{61.1} \\
\hline \multicolumn{4}{|c|}{11.1} \\
\hline \multicolumn{4}{|c|}{22} \\
\hline \multicolumn{4}{|c|}{22} \\
\hline \multicolumn{4}{|c|}{16.6} \\
\hline \multicolumn{4}{|c|}{61.1} \\
\hline \multicolumn{4}{|c|}{88.9} \\
\hline \multicolumn{4}{|c|}{11.1} \\
\hline \multicolumn{4}{|c|}{61.1} \\
\hline & \multicolumn{2}{|c|}{38.8} & \\
\hline Mínimo & Máximo & Media & $\begin{array}{l}\text { Desviación } \\
\text { estándar }\end{array}$ \\
\hline 0.6 & 18 & 10.7 & 5.05 \\
\hline 0.2 & $\underline{4.8}$ & 1.8 & $\underline{1.2}$ \\
\hline 19 & 69 & 38.8 & 11.6 \\
\hline 0.11 & 13 & 2.4 & 2.9 \\
\hline
\end{tabular}

Fuente: datos obtenidos del estudio y analizados en SPSS. 2018 
De este análisis emergieron seis temas: 1) idealización del niño fallecido; 2) batalla perpetua; 3) vínculo inquebrantable; 4) pérdida de sentido; 5) levantarse y seguir, y 6) lucha espiritual. Cada uno de los temas se describe a continuación:

\section{Idealización del niño}

Los cuidadores describen a los niños como personas que fueron activas, amorosas, guerreras y ejemplares, quienes a su corta edad habían atravesado por experiencias difíciles con su salud, lo que les hacía apreciar aspectos de la vida que un niño en otra sitación no comprendería. Además, tenían un cierto grado de madurez para afrontar el tratamiento y la enfermedad, además de tener otras perspectivas de la vida que los hacía ser buenos consejeros.

La vida de A. fueron 7 años larguitos [...] y en esos 7 años le enseñó a mucha gente cosas que yo en 36 años no les he enseñado porque a los 7 él hablaba con madurez, era sincero; entonces, si él sabía tanto, hay que tener fe, hay que creer que se puede (Participante 4).

Los padres evocan recuerdos sobre lo que eran los niños y describen el comportamiento que tenían, sus gestos de afecto y las anécdotas sobre su forma de ser; consideran que los niños representaban la aventura de su vida y que sus actitudes mostraban la fortaleza que tenían mientras sobrellevaban la enfermedad, además de su constante disposición para ayudar a otros: “[...] Él llegaba y era el que estaba pendiente de todo lo que fuera; que toma esto, que la limonada, que los zapatos, que las chanclas, que la camisa, que ... lo que fuera; él estaba pendiente de cualquiera" (Participante 6).

En general, los padres expresan esas características especiales de los niños, las cuales los llevaban a pensar en un aspecto sobrenatural que los hacía diferentes, lo que les daba la ilusión de creer que eran seres superiores que les habían brindado la oportunidad de aprender algo importante sobre la vida y que esa enseñanza era su misión.

\section{Batalla perpetua}

La vivencia de los padres durante la enfermedad y la muerte de sus hijos es una lucha que para ellos permanece a lo largo de la vida. Los padres describen que no pudieron volver hacia atrás o tomar otras decisiones que cambiaran la situación de enfermedad del niño, y lo dicen haciendo referencia a los múltiples tratamientos que no fueron eficaces y que se convirtieron en una lucha interminable, en la que todo el esfuerzo y el dolor por el que atravesaron fueron infructíferos.

Pero, ya en los límites, pues, eso no tiene cura. ¿Por qué hace uno tratamiento? Si es que con eso no se puede, eso queda como darle un tiempo y vuelve y aparece, y vuelve y arranca, y arranca como más fuerza; como que no, eso con el cáncer no se puede (Participante 2).

Sin embargo, para estos padres la batalla continúa, incluso después de la muerte del niño. Se enfrentan a la dualidad entre la sensación de no poder seguir con la vida versus la situación del mundo real, en el que existen gastos y se debe trabajar. Advierten que hablar es indispesable como proceso para ayudarse a sí mismos a sanar y a continuar con la vida, además de intentar reorganizarse después de la muerte, lo que ellos describen como un ajuste a la normalidad.

Pues duro, pero pues igual, tocaba seguir de igual porque trabajábamos en una empresa y de igual manera no podíamos quedarnos sin el trabajo porque el dinero nos hacía falta, o sea, siempre nos tocó conservar eso de cualquier manera. Al comienzo pues muy duro, uno casi que no quiere, no sabe cuál rumbo tomar, pero pues después le toca a uno situarse y asumir las cosas. Entonces fue cómo lo que hicimos todos, de igual manera, seguimos a nuestro ritmo porque no había otra manera (Participante 6).

O en palabras de otro participante: "Como volviendo todo otra vez a la normalidad. O sea, uno piensa que eso es como un sueño, que fue como un sueño y que pues Dios quiere y nunca vuelva a pasar" (Participante 3).

Así, la batalla librada por el cuidador se percibe como eterna, ya que no solo fue el proceso de la enfermedad y el tratamiento por el cual pasó el niño, sino lo que representa su pérdida, el momento de la vida en el cual luchan a diario con los sentimientos de duelo y los recuerdos del niño, y se evidencia lo difícil que es para ellos continuar con su vida normal. 


\section{Vínculo inquebrantable}

La experiencia del cuidado del niño durante la enfermedad y la muerte genera un vínculo que es inquebrantable para los cuidadores; así, cuando ocurre la muerte del niño, se genera en ellos un vacío que persiste en el tiempo con la sensación de estar incompletos, de no tener una presencia física con la cual relacionarse; sin embargo, al mantener los recuerdos, las cosas y los espacios en los que compartían con el niño, hacen que esta unión trascienda y le asigna significado a la experiencia.

Y ahora que ya no está, nosotros hablábamos eso. Ella se fue pero el amor siguió, ese sentimiento que había hacia ella y que uno sentía que ella tenía hacia nosotros todavía se siente, no es que se murió y ya, entonces todo murió, no. Por eso es que el amor verdadero trasciende hasta la muerte (Participante 10).

Para estos padres vivir la enfermedad con sus hijos representó no solo sufrimiento, sino un proceso de aprendizaje único e irrepetible, en el que emergieron sensaciones y sentimientos de amor incondicional que junto con su presencia auténtica hizo aún más significativa la conexión. Así mismo, los cuidadores consideran que la unión entre padre e hijo trasciende a lo espiritual; su creencia en la existencia de una vida después de la muerte les permite pensar que su espíritu permanece con ellos o, incluso, mantienen la esperanza de un reencuentro al momento de morir.

\footnotetext{
Algo, o sea, algo que uno nunca debe dejar es lo que vivió, y las enseñanzas que uno tuvo de eso y que le quedan a uno; y que uno sabe que esa persona siempre estará con uno, o sea, ese cariño y ese afecto hacia esa persona nunca cambiará así lleguen más cosas; son nuevas cosas, son amores muy diferentes a todos (Participante 10).

Uno lo siente, porque yo tengo en la mente las últimas palabras que ella me dijo: - Búscame ahí en tu corazón, que siempre voy a estar contigo mamita. Te voy a llenar siempre de besos y siempre estoy contigo (Participante 5).
}

Este vínculo del cuidador con el niño hace que el proceso de duelo sea complejo, pero no imposible de sobrellevar; de manera que, con el paso del tiempo, los padres logran despedirse de sus hijos y de la experiencia negativa que tuvieron con ellos, perpetuando los buenos recuerdos del niño y el aprendizaje obtenido de esa situación.

\section{Pérdida de sentido}

Las dificultades internas e interpersonales de los cuidadores para reacomodarse después de la muerte del niño derivan en la pérdida de sentido para seguir con la vida; no encuentran razones ni motivos para continuar con lo que están haciendo o retomar lo que hacían antes de la enfermedad del niño. Esta sensación se acompaña de sentimientos de ira, miedo, cansancio, culpa, frustración, impotencia e incertidumbre ante lo acontecido, y ante lo que está por venir. Lo anterior forma parte del proceso normal de duelo; sin embargo, se convierte en algo nocivo cuando la persona no logra manejarlos o sobreponerse a ellos.

El proceso de la pérdida fue...Es realmente, fue cómo...es algo que uno no sabe explicar, es algo que duele, es algo que le hace sentir culpable, qué le hace sentir impotente, qué le hace sentir temor, no sé, que lo hace sufrir de todas maneras a uno, y es terrible (Participante 13).

Muchas de las maneras de afrontar la muerte del niño por parte de los cuidadores se evidencian en actitudes de agresividad ante pequeños problemas, aislamiento social, autolesiones físicas e, incluso, alcoholismo, ideación e intento de suicidio y autoabandono. Estos hechos demuestran la dificultad para enfrentar la pérdida y las medidas poco efectivas para manejar sus emociones, pues sienten que, con la muerte del niño, simbólicamente mueren ellos también.

Yo abandoné el trabajo, mi hermano abandonó el trabajo, es que todo el mundo estaba loco. Y eso con el paso de los días ellos no, mi hermano ya no podía, y no, y a él la vida le importa cinco ahora... él todos los días toma, él todos los días llora, él todos los días llega al cementerio, él allá se planta, en la tumba (Participante 2).

\section{Levantarse y seguir}

Con el paso del tiempo, los cuidadores comprenden que la vida continúa y, con el apoyo de su familia y otras personas cercanas, logran encontrar la forma de crecer en esta experiencia, de aprender 
significativamente de ella y llegar a la aceptación. No se debe desconocer que, para levantarse y seguir, los cuidadores necesitan apoyo emocional y ayuda profesional, sumado a una guía espiritual y a los grupos de apoyo de cuidadores que han pasado por situaciones similares.

Yo, a pesar de que él falleció un viernes, y el domingo lo enterré, a los ocho días ya me fui a trabajar; no hubo descanso porque para mí la casa era terrible. Mi esposo era muy encima y no me dejaba sola; entonces traté como de salir, de distraerme de todo, pero si ha sido difícil (Participante 7).

Sí, uno aprende de esto... la pérdida de mi hijo y su enfermedad me ayudaron a estar un poquito más concentrada con mis otros hijos, que así ya sean más viejos, o esto, pero como a estar un poquito más pendientes de ellos, que de pronto una comida, que camine vamos allí a tal lado, que un te amo, pues yo si soy cariñosa, pero sí muchas veces se concentra más uno en sí, en trabajar, en la rutina (Participante 17).

Seguir y levantarse es un proceso diario y paulatino que requiere de la ayuda del entorno del cuidador, además de una reflexión propia en la que se utilicen estrategias para entender lo sucedido: aprender de la experiencia y retomar aquello que no habían podido hacer. En algunos casos, estos padres se dan la oportunidad de tener otro hijo luego de este acontecimiento, viéndolo como una nueva oportunidad; incluso afirman que con la llegada de este bebé se mitiga algo el dolor: "Pues una de las ventajas que nosotros tenemos fue haber tenido a S. Que ella haya nacido, porque nos ayudó mucho a... pues no ocupa el lugar de mi niño pero sí, uno a la vez piensa que es él también. ¿Sí? Que está ahí en ella" (Participante 3).

\section{Lucha espiritual}

Con la muerte de los niños, los cuidadores intentan buscar explicaciones a esta situación, de manera que, al no tener razones lógicas y tangibles, recurren a su fe en un ser superior, lo que les otorga sentido a eso que está más allá de su comprensión. Acercarse a Dios en busca de solución, consuelo y fortaleza ha sido una de las vías para afrontar la muerte, pero no sin antes cuestionarse sobre Dios y su justicia divina.
Eso me dijeron los médicos cuando me dieron la última respuesta de la niña, que ya no había nada que hacer, ya se le iba de las manos, que ellos no entendían por qué, si no se les había perdido ni un control, ni quimioterapias, ni nada... Y nosotros no entendemos por qué si es hija única y es una niña tan inteligente, mi Dios nos la dio; nosotros no entendemos la ciencia médica, sobre Dios ya no se puede hacer más. (Participante 14)

La lucha espiritual de los cuidadores se da como consecuencia de tener una relación ambigua con Dios, porque, pese a cuestionar su fe en lo acontecido, los padres continúan recurriendo a este ser superior por medio de la oración, pidiendo fortaleza para seguir con su vida y para que el niño esté en paz donde se encuentre. Así mismo, perciben algunos cambios positivos en su estado de ánimo como una respuesta de Dios, y con el tiempo trascienden emocional y espiritualmente, al ver la muerte como un paso natural que forma parte de la vida.

Esa enfermedad es tan terrible que uno le pregunta al Señor: ¿me tengo que ir?, ¿por qué con ella?, ¿por qué nos hace eso?, ¿por qué nos abandona?, ¿Es que usted no existe? ¿por qué es tan injusto? y uno pierde la fe (Participante 2).

Me hizo sentar a pensar, cuando me dijeron que la niña estaba sufriendo y que yo no podía ser egoísta, que el tiempo de Dios sería perfecto; que... pues, lo que Dios quería era para bien; entonces, pues yo no sé, como que empecé a tratar de entender... y como que eso me fue calmando (Participante 13).

\section{Discusión}

A través de la descripción de las experiencias de los cuidadores con la pérdida de un niño con cáncer, se evidencian las percepciones, sensaciones y sentimientos que esta difícil vivencia deja en ellos; y, a pesar de lo dolorosa que pueda ser, evidencia un proceso de duelo que les ha dejado enseñanzas y les ha permitido comprender la trascendencia de pensar en una vida después de la muerte.

Según Alameda y Barbero (18), el duelo por la muerte de un hijo tiene un carácter antinatural debido al valor del vínculo entre padres e hijos, sumado al pensamiento de que existe un orden natural en el cual los padres mueren primero que

- S. Carreño-Moreno - L. Chaparro-Díaz - R. López-Rangel 
los hijos. La pérdida de un hijo genera un profundo dolor, así como desconcierto e incertidumbre con las expectativas respecto al futuro. Estos argumentos se ven reflejados en las descripciones de los cuidadores, en las que expresan tristeza, a pesar del paso del tiempo, además de la dificultad para retomar nuevamente su vida cotidiana.

La muerte de un miembro de la familia (como lo es un hijo) genera todo tipo de disfuncionalidades en los cuidadores. En este caso, las expresiones de los participantes muestran algunos de los problemas que se pusieron en pausa mientras transcurría la enfermedad del niño y que se reavivan con su muerte. Así, algunos autores $(4,18)$ sustentan que las vivencias que cada miembro de la familia ha tenido con el fallecido influirán en el duelo, tanto a nivel individual como familiar, y que su resolución depende de la capacidad para solucionar esos conflictos internos e interpersonales.

Frente a las implicaciones de este tema, diversos autores $(18,5,27)$ han descrito los efectos físicos, emocionales, sociales y espirituales que conlleva el duelo por la muerte de un hijo. Los estados de ánimo, por ejemplo, pueden variar y manifestarse con distintas intensidades; predominan sentimientos como la tristeza, la rabia, la culpa, el miedo, la impotencia, la añoranza, el abatimiento, el alivio y la liberación, lo cual coincide con lo expresado por los cuidadores, en especial al inicio del proceso de duelo por la muerte del niño.

Al sufrir la pérdida, los cuidadores tienden hacia conductas inadecuadas o que nunca antes habían tenido, se convierten en personas inactivas, aumentan el consumo de tabaco, alcohol $\mathrm{y} / \mathrm{u}$ otro tipo de sustancias, sumado al aislamiento social $(18,5,27)$. Estos son aspectos que describen las vivencias de los participantes en el tema denominado pérdida de sentido, en el cual los cuidadores muestran las situaciones que atravesaron y las decisiones que tomaron sobre su propia vida en un momento posterior a la muerte del niño. Las sensaciones, sentimentos y reacciones dadas en ese periodo de tiempo corresponden a una fase inicial de duelo en el que prevalece la negación, el enfado, la indiferencia y la toma de decisiones que afectan negativamente la vida de la persona (28).
Dentro de la práctica, los profesionales de la salud deben pensar en intervenciones para estos cuidadores, en las que prime una presencia auténtica y un acompañamiento durante el tiempo en que los niños están en fase de cuidados paliativos; como equipo interdisciplinar se debe trabajar en la incertidumbre ante el futuro, la aceptación de la cercanía de la muerte y el reconocimiento de los problemas familiares preexistentes, con el fin de darle a los padres varios caminos a seguir luego del fallecimiento del niño.

En cuanto a la dimensión espiritual, todos los cuidadores son creyentes en un ser superior. En este caso, Dios es el ser en el que se refugiaron, tanto para buscar fortaleza como para encontrar una explicación a esta situación. Adicionalmente, transitar por esta experiencia les permitió comprender y aceptar que en cada uno de ellos queda el ser del niño, no su presencia física pero sí la presencia espiritual. Esta percepción de los padres es respaldada por Riley ET AL. (29) y Kelley y Chan (30), quienes plantean que es en lo espiritual donde se cuestionan y buscan respuestas sobre el sentido de la vida, el planteamiento de las propias creencias y la idea de trascendencia.

Para Montoya (31), el hecho de ser el cuidador principal también afecta la forma en la que percibe lo que sucede. Para algunos, la muerte del niño puede representar un alivio, pues este deja de sufrir. Esta concepción se hace evidente en los participantes, especialmente en el tema denominado levantarse y seguir, y se corresponde con fases más avanzadas del duelo, que pueden llegar incluso a la aceptación.

Los padres que han perdido un hijo se enfrentan a dos retos: 1) aprender a vivir sin el niño, que encarnaba una gran proyección de futuro, y 2) interiorizar una representación interna del niño que le sirva de consuelo $(32,33)$. En esta investigación, los cuidadores describen que prefieren mantener buenos recuerdos del niño, pensar en los momentos agradables que pasaron y no en el sufrimiento que representó la enfermedad; a pesar de ello, no dejan de sentirse frustrados porque, para ellos, el tiempo no fue suficiente para realizar todos los planes que tenían con y para sus hijos. 
De acuerdo con Peiró, Corbellas y Blasco (34), recordar al fallecido sin una intensidad exagerada es un indicador de que el duelo acaba; pero cuando el duelo dura demasiado, o se estanca en alguna fase impidiendo el desarrollo normal de la vida, se hace indispensable la ayuda de un profesional. Es aquí donde los profesionales de la salud deben actuar. Conocer la experiencia de estos cuidadores permite entender las percepciones acerca de esta vivencia, actuar de manera empática y promover la comunicación de sentimientos.

En general, las implicaciones prácticas de los resultados de este estudio deben reorientrar los servicios de salud especializados en los cuales se maneja cuidado paliativo y donde el foco no solo sea el paciente sino también el cuidador. Efectivamente, compartir la experiencia de la pérdida en un ambiente nutritivo y humanizado promueve formas de trabajar en el dolor, la rabia y la frustración, por lo que construir y favorecer redes y grupos de apoyo con otros padres en la misma situación y con el equipo de salud que cuidó del niño es una acción concreta pero trascendental para la elaboración del duelo $(35,36,37)$.

Por último, es necesario reconocer que, para futuras investigaciones, es fundamental identificar los factores que favorecen un adecuado proceso de duelo; conocer las motivaciones de los padres para seguir adelante y la forma en que el soporte social ayuda a dar significado a la experiencia; además, ante la inminente cercanía a la muerte e incluso dentro del proceso de cuidado y tratamiento del niño con cáncer, es importante indagar por la toma de decisiones compartidas entre padres y profesionales de la salud.

\section{Conclusiones}

Los seis temas denominados como idealización del niño fallecido, batalla perpetua, vínculo inquebrantable, pérdida de sentido, levantarse y seguir, y lucha espiritual, los cuales fueron descritos, evidencian las reacciones habituales y comunes en este tipo de duelo, de manera que no se puede dejar de lado que este es un proceso por el que pasará el cuidador hasta lograr la adaptación a la realidad y permitirse trascender la ausencia física del niño.
Con el duelo la persona necesita una adaptación constante para mantener el equilibrio personal y una calidad de vida aceptable. Para conseguir este objetivo, el cuidador necesita del apoyo del profesional de enfermería y de otras disciplinas que le permitan entender sus sentimientos y emociones, idear estrategias para su manejo y finalmente aceptar la situación.

En síntesis, conocer la experiencia del cuidador familiar ante el duelo del niño fallecido por cáncer se constituye en un aporte para el conocimiento, porque permite ampliar lo ya conocido acerca de algunas características del duelo en los cuidadores, además de complementar aspectos como las necesidades, creencias, mecanismos de afrontamiento e interacciones significativas que dan a los profesionales de la salud herramientas para ayudar al cuidador a reconstruir el sentido de su vida.

\section{Referencias bibliográficas}

1. Organización Mundial de la Salud. International Childhood Cancer Day: 15 February 2018 [Internet]; 2018. Disponible en: https://www.who.int/cancer/iccd-2018/en/

2. Piñeros M, Gamboa $\mathrm{O}$, Suárez A. Mortalidad por cáncer infantil en Colombia durante 1985 a 2008. Rev Panam Salud Pública. 2011;30(1): 15-21.

3. Jalmsell L, Kontio T, Stein M, Henter JI, Kreicbergs U. On the child's own initiative: parents communicate with their dying child about death. Death studies. 2015;39(2): 111-117. https://doi.org/10.1080/07481187.2 014.913086

4. Alam R, Barrera M, D'Agostino N, Nicholas DB, Schneiderman G. Bereavement experiences of mothers and fathers over time after the death of a child due to cancer. Death Studies. 2012;36(1): 1-22. https://doi.org /10.1080/07481187.2011.553312

5. Vega P, Rivera S, González R. El transitar del sobrevivir al revivir: comprender la vivencia de padres y madres que han perdido a sus hijos a causa del cáncer. Rev Chil Ped. 83(3);247-57. https://doi.org/10.4067/S0370-41062012000300006

6. García V, Rivas E. Experiencia de enfermeras intensivistas pediátricas en la muerte de un niño: vivencias, duelo, aspectos bioéticos. Cienc Enf. 2013;19(2):111-24. https://doi.org/10.4067/S0717-95532013000200011 
7. Dokken D. Making meaning after the death of a child: bereaved parents share their experiences. Pediatr Nurs. 2013;39(3):147-50.

8. Rosenberg AR, Dussel V, Kang T, Geyer JR, Gerhardt CA, Feudtner C, Wolfe J. Psychological Distress in Parents of Children With Advanced Cancer. JAMA Pediatr. 2013;167(6):537-543. https://doi.org/10.1001/ jamapediatrics.2013.628

9. Yoshida S, Amano K, Ohta H, Kusuki S, Morita T, Ogata A, Hirai K. A comprehensive study of the distressing experiences and support needs of parents of children with intractable cancer. Jpn J Clin Oncol. 2014;44(12):1181-8. https://doi.org/10.1093/jjco/ hyu140

10. Barreto P, Yi P, Soler C. Predictores de duelo complicado. Psicoonc. 2008; 5(2-3): 383-400.

11. Correa IC. Duelo por pérdida de un hijo(a): historia de vida de padres y madres de la Fundación Lazos Medellín. EN-Clave Social. 2013; 2(2): 70-78.

12. Kersting A, Brähler E, Glaesmer H, Wagner B. Prevalence of complicated grief in a representative population-based sample. Journ Affect Disord. 2011; 131(1-3): 339-343. https://doi.org/10.1016/j.jad.2010.11.032

13. Maccallum F, Galatzer IR, Bonanno GA. Trajectories of depression following spousal and child bereavement: A comparison of the heterogeneity in outcomes. Journ Psych Res. 2015;69:72-79. https://doi.org/10.1016/j. jpsychires.2015.07.017

14. Colombo G. Estilos de afrontamiento y su relación con el estrés en padres con hijos menores de edad con diagnóstico de cáncer. Psocial. 2018;4(1):82-94.

15. Navea A, Tamayo JA. Características de la resiliencia familiar en pacientes oncológicos pediátricos: una revisión sistemática. Psicooncología. 2018;15(2): 203-216. https://doi.org/10.5209/PSIC.61431

16. Cacciatore J, Froen F, Killian M. The effects of self-blame on anxiety and depression among women who have experienced stillbirth. Journ Ment H Couns. 2013;35(4): 342-359. https://doi.org/10.17744/mehc.35.4.15427g $822442 \mathrm{~h} 11 \mathrm{~m}$

17. Stroebe M, Stroebe W, Van de Schoot R, Schut H, Abakoumkin G, Li J. Guilt in bereavement: the role of self-blame and regret in coping with loss. PloS One. 2014; 9(5): e96606. https://doi.org/10.1371/journal. pone.0096606

18. Alameda A, Barbero J. El duelo en padres del niño oncológico. Psicooncología. 2009; 6(2-3):485-498.

19. Cruz-J de J. El concepto de experiencia en Victor W. Turner, E. P. Thompson y Anthony Giddens: Un diálogo entre antropología social, historia y sociología. Soc Hist. 2017;(7):345-375.

20. Colaizzi PF. Reflection and research in psychology; a phenomenological study of learning. Dubuque, Iowa, Kendall/Hunt Pub. Co; 1973.

21. Hernández R, Fernández C, Baptista P. Metodología de la Investigación. Mexico: MacGraw-Hill; 2014.

22. Noreña A, Alcaraz N, Rojas J, Rebolledo D. Aplicabilidad de los criterios de rigor y éticos en la investigación cualitativa. Aquichan. 2012;12(3). https://doi. org/10.5294/aqui.2012.12.3.5

23. Colaizzi PF. Psychological research as the phenomenologist views it. En: Ronald S. Valle y Mark King (eds.), Existential-Phenomenological Alternatives for Psychology. New York: Oxford University Press; 1978. p. 48-71.

24. Guba EG, Lincoln YS. Competing paradigms in qualitative research. Handb Qual Res. 1994;2(163-194):105.

25. Consejo De Organizaciones Internacionales (Cioms y OMS). Ethical Guidelines for Biomedical Research on Human Beings. Geneva; 2002.

26. Congreso de la República de Colombia. Ley 911 de 2004. Colombia: Diario Oficial No. 45.693;2004.

27. Gilmer MJ, Foster TL, Vannatta K, Barrera M, Davies B, Dietrich MS ET AL. Changes in parents after the death of a child from cancer. Journ Pain Symptom Manag. 2012; 44(4):572-82. https://doi.org/10.1016/j.jpainsymman.2011.10.017

28.Pascual AM, Santamaría JL. Proceso de duelo en familiares y cuidadores. Rev Esp Geriatr Gerontol. 2009;44(S2):48-54. https://doi.org/10.1016/j. regg.2009.05.012

29. Riley, LP, La Montagne LL, Hepworth JT, Murphy BA. Parentalgriefresponses and personals growth following the death of a child. Death Studies. 2007;31(4):277-299. https://doi.org/10.1080/07481180601152591

30. Kelley MM, Chan KT. Assessingthe role of attachment to God, meaning, and religious coping as mediators in the grief experiencia. Death Studies. 2012;36(3):199227. https://doi.org/10.1080/07481187.2011.553317

31. Montoya J. Pérdida, aflicción y luto: manual para la recuperación. México: Trillas; 2012.

32. Brandstätter M, Kögler M, Baumann U, Fensterer V, Küchenhoff H, Borasio GD, Fegg MJ. Experience of meaning in life in bereaved informal caregivers of palliative care patients. Sup Care Cancer. 2014;22(5): 1391-9. https://doi.org/10.1007/s00520-013-2099-6 
33. Correa M, Granero J, Hernandez JM, Fernandez C. Transferring palliative-care patients from hospital to community care: A qualitative study. At Prim. 2016; 49(6), 326-34. https://doi.org/10.1016/j. aprim.2016.09.003

34. Peiró G, Corbellas C, Blasco A. El duelo en la pérdida de un hijo. En: Camps C, Sánchez PT, eds. Duelo en Oncología. Madrid, España: Sociedad Española de Oncología Médica; 2007. p. 85-101.

35. Endo K, Yonemoto N, Yamada M. Interventions for bereaved parents following a child's death: A systematic review. Palliat Med. 2015;29(7):590-604. https://doi.org/10.1177/0269216315576674

36. Foster TL, Gilmer MJ, Davies B, Dietrich MS, Barrera M, Fairclough DL. Comparison of continuing bonds reported by parents and siblings after a child's death from cancer. Death Studies. 2011;35(5):420-40 https:// doi.org/10.1080/07481187.2011.553308

- S. Carreño-Moreno - L. Chaparro-Díaz - R. López-Rangel 